\title{
Ovarian Ectopic Pregnancy with Newborn at Term: A Case Report
}

\author{
Müller $\mathbf{R}^{1}$, Quirino ${ }^{1 *}$ and Quintairos $\mathbf{R}^{2}$ \\ ${ }^{1}$ Fundação Santa Casa de Misericórdia do Pará, Brazil \\ ${ }^{2}$ Hospital Porto Dias, Brazil
}

Submission: June 05, 2018; Published: July 16, 2018

*Corresponding author: Quirino I, Fundação Santa Casa de Misericórdia do Pará, 1040,Rua Bernal do Couto. Belém, Brazil, Tel: +559140092200; Email: ivyqds@gmail.com

Keywords: Ovarian pregnancy; Ectopic pregnancy; Non-tubal ectopic pregnancy; Circulatory collapse; Wedge resection; Radical; Oophorectomy; Patients; Scotoma; Kidney; Epigastralgy; Ultrasonography; Hemorrhage; Thromboembolism; Urinary tract; Intrauterine growth; Placental; Lysis of adhesion; Ovarian gestation; Fetal vitality; Local vascularization

\section{Introduction}

Ovarian pregnancies represent between 1 to $6 \%$ of all ectopic pregnancies and remain a challenge for the diagnosis [1], even with technological advancements in imaging methods. It is estimated that this event occurs in up to 1:70.000 pregnancies and one of the consequences is the rupture at an earlier stage, associated with high rates of circulatory collapse and maternal fetal morbidity and mortality [2,3]. In relation to the ovaries, when diagnosed in early stages, most of them require surgical treatment; it can be conservative (wedge resection) or radical (oophorectomy). The first option can be destinaded to patients with reproductive desire or single ovary, and the second is limited to cases of advanced pregnancies. The definitive diagnosis is made during the intraoperative period and histopathological confirmation [4]. The placenta may be left in situ in situations involving large vessels or vital structures, as spontaneous resorption will occur. Placental maintenance is associated with great morbidity such as paralytic ileus, intestinal obstruction, thromboembolism, hemorrhage or infections, however, low mortality [5].

\section{Case Report}

E.M.P., 26 years old, black, married, primigravida, admitted to the service at 28 weeks and 1 day of gestation, with mild pain in the lower abdomen and blood pressure $170 \times 120 \mathrm{mmHg}$, without headache, scotoma or visual turbidity. She underwent this first hospitalization for clinical investigation, with a diagnostic hypothesis of Hypertensive Disease of Gestation and nephropathy to be clarified. She was submitted to complementary examinations with ultrasound of urinary tract that diagnosed a single kidney. The conclusion of the obstetric ultrasound was single fetal in pelvic presentation, longitudinal situation, gestational age of 30 weeks and 4 days, estimated weight of $1528 \mathrm{~g}$.

The patient was submitted to a complementary transvaginal US, where it was observed in right adnexal region uterus image, measuring $12 \mathrm{~cm}$ in the largest diameter with $10 \mathrm{~mm}$ endometrium and empty cavity. A fetus was identified on the right flank and the hypotheses of topic pregnancy (uterus Didelphys) or ectopic pregnancy (abdominal pregnancy) were suggested.Magnetic resonance imaging of the pelvis revealed Mullerian malformation with the presence of two uterine bodies, single cervix and norm implanted placenta. One of the cavities had a fetus in pelvic presentation, while the other had no alterations. After the examination, it was determined that there was no urgency to resolve the gestation and the patient was discharged after the stabilization of the clinical condition.

The patient was readmitted after 8 days complaining of lower abdominal pain, epigastralgy and blood pressure of 170x120mmHg. She had an obstetric ultrasonography performed indicating a single, live fetus in pelvic presentation, restriction of intrauterine growth below p10, with gestational age of 32 weeks and 3 days, weight of $1860 \mathrm{~g}$. The concept evolved with acute fetal distress and bradycardia, and the cesarean section was indicated. During the surgery, extra-uterine gestation was identified, and the gynecological surgery team was informed.Placental and fetal insertion was detected in the right ovary (Figure 1) with intense vascularization coming from the abdominal aorta (Figure 2). An intense adhesion process of the omentum and bowel were also identified, as well as the left ovary without alterations and a nongravid uterus with normal format and consistency. 


\section{Global Journal of Reproductive Medicine}
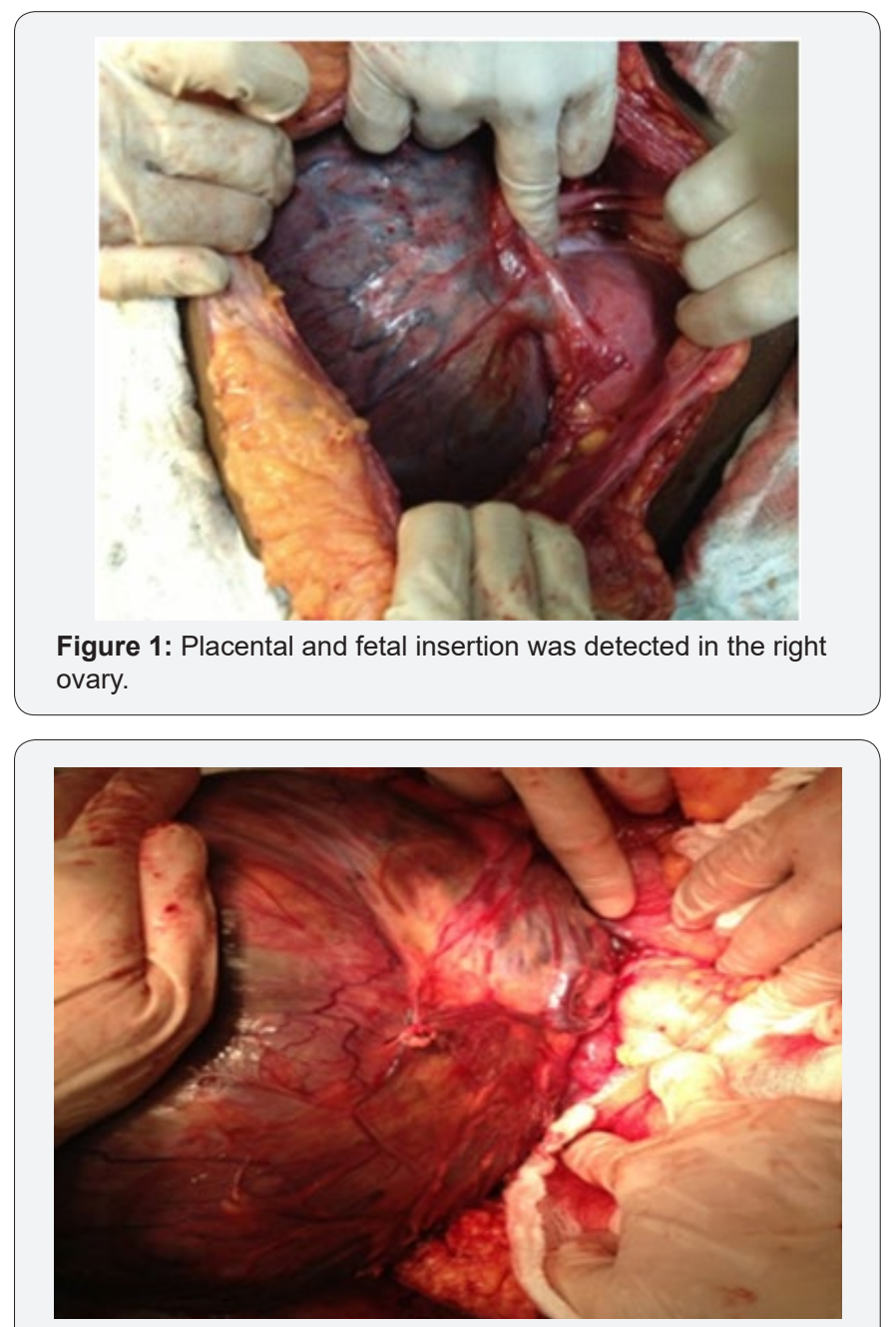

Figure 2: vascularization coming from the abdominal aorta.

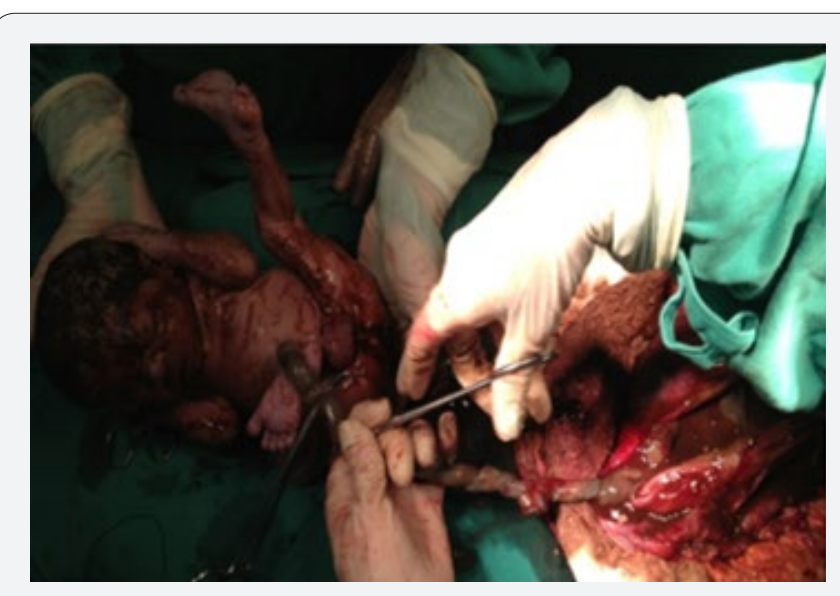

Figure 3

After lysis of adhesion, the extraction of a single live concept, with 1715g of weight, APGAR 8/9, plated in thick meconium fluid and Capurro of 37 weeks was performed (Figure 3). The cord was clamped at the placental insertion base and the ovary was sutured using a synthetic absorbent thread and applying regenerated oxidized cellulose to prevent pelvic adhesions in the future (Figure 4). At the end of the procedure, she was referred to the ICU, prescribed prophylactic antibiotics, analgesia and prophylaxis for thromboembolism. After 3 days, the patient was referred to the highrisk ward, which underwent infectious, hemantimetric and imaging tests to prevent acute hemorrhagic disease. Placental resorption was monitored for a period of 2 months and after stabilization of the condition, the patient was referred to the specialty outpatient clinic.

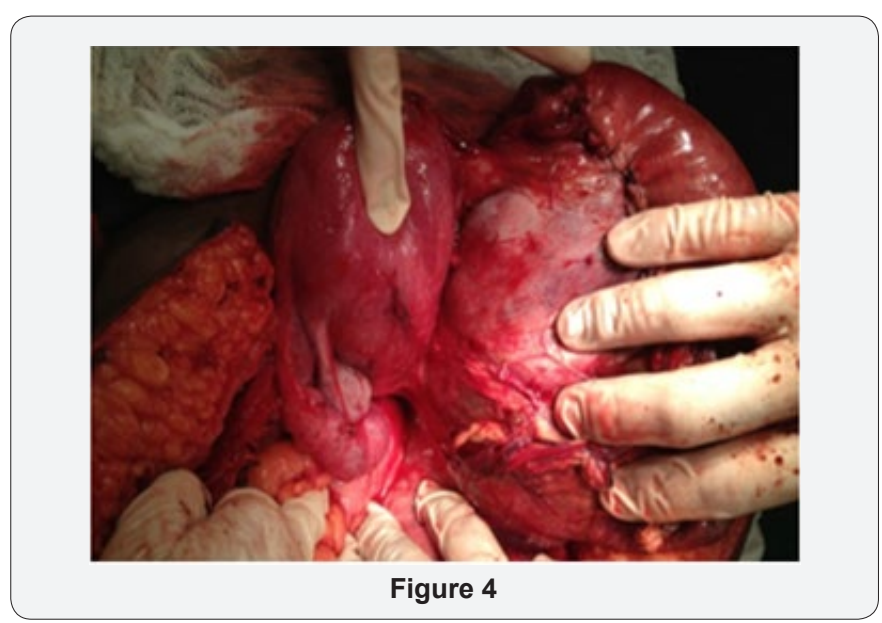

\section{Discussion}

Ovarian gestation is a rare occurrence in obstetrics, becoming an even rarer event when it progresses with a live, viable fetus until its birth and with postnatal development without alterations [6]. The case in question demonstrates an immense diagnostic challenge, even with the use of nuclear magnetic resonance, since it is a rare obstetric pathology. The favorable evolution observed in the report, with survival of the mother and its concept, constitutes the exception, not the rule. Therefore, we must continue to follow the recommendations of the latest evidence, acting with the interruption of gestation treatment facing an early diagnosis, through clinical or surgical management $[7,4]$. When the diagnosis occurs between the second and third trimester, due to an intense local vascularization, it is possible to opt for an expectant conduct and control of the fetal vitality until it reaches the viability. Although we must explain all the risks and leave the patients comfortable to decide whether they will choose or not to continue the pregnancy and receive the signed informed consent.The guidelines of greater impacts guide the interruption of pregnancy, either by clinical or surgical methods. Therefore, based on the case presented and some other rare reports in the literature, we are faced with the need for more publications that may guide us to maintain an ovarian pregnancy until viability or follow the current recommendations.

\section{References}

1. Shen L, Fu J, Huang W, Zhu H, Wang Q et al. (2014) Interventions for non-tubal pregnancy. Cochrane database of systematic reviews.

2. Birge O, Erkan MM, Ozbey EG, ArlanD (2015) Medical management of an ovarian ectopic pregnancy: a case report. J Med Case Rep 9: 290.

3. Melcer Y, Smorgick N, Vaknin Z, Mendlovic S, Raziel A, et al. (2015) Primary Ovarian Pregnancy: 43 Years experience in a single institute and still a medical challenge. IMAJ 17: 687-690. 
4. Samara N, Bentov Y (2016) Case Report of ectopic ovarian pregnancy following fresh embryo transfer. Clin Med Insights Reprod Health10: 29-32.

5. (2016) Diagnosis and management of Ectopic Pregnancy. Green-top Guideline No. 21. RCOG/AEPU. Joint Guideline.

6. Ranaivoson HV, Ranaivomanana VF, Nomenjanahary L, Andriamampionona TF, Randrianjafisamindrakotroka NS (2016)
Grossesse ovarienne:à propos de 3 cas et une revue de la literature. Pan Afr Med J 25: 128.

7. (2012) Ectopic pregnancy and miscarriage: diagnosis and initial management. National Institute for Health and care Excellence. Clinical guideline.

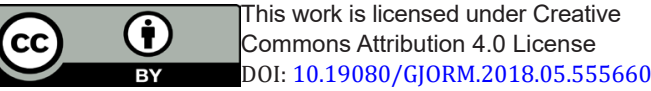

